\title{
Erratum
}

\section{Investigation of heteroepitaxial diamond films by atomic force and scanning tunneling microscopy}

\author{
K. Schiffmann, X. Jiang \\ Appl. Phys. A 59, 17-22 (1994)
}

In this contribution, several wrong numerical values for the crystal tilt have been published in the text on pages 17, 20, 22, and in Fig. 5a-d on page 22.
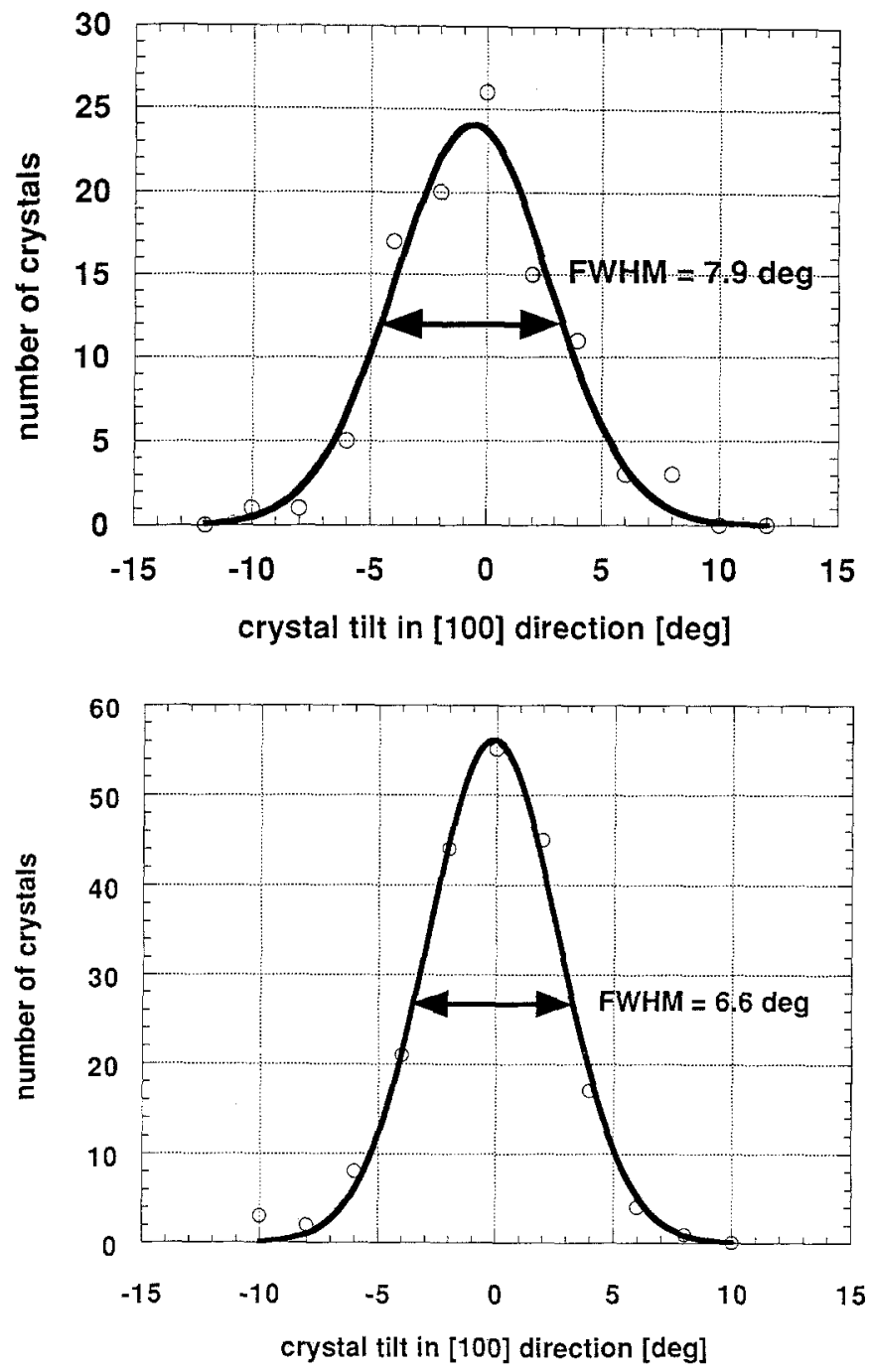

Fig. 5a-d. Distributions of tilt angles of the (001) crystal surface of heteroepitaxial diamond crystallites relative to its silicon substrate. (a) and (b): tilt for sample 1 in [100] and [110] direction;
Page 17:

Besides the good macroscopic crystal morphology a statistical tilt up to $\pm 5.2^{\circ}$ of the oriented crystallites has been found relative to the silicon substrates.
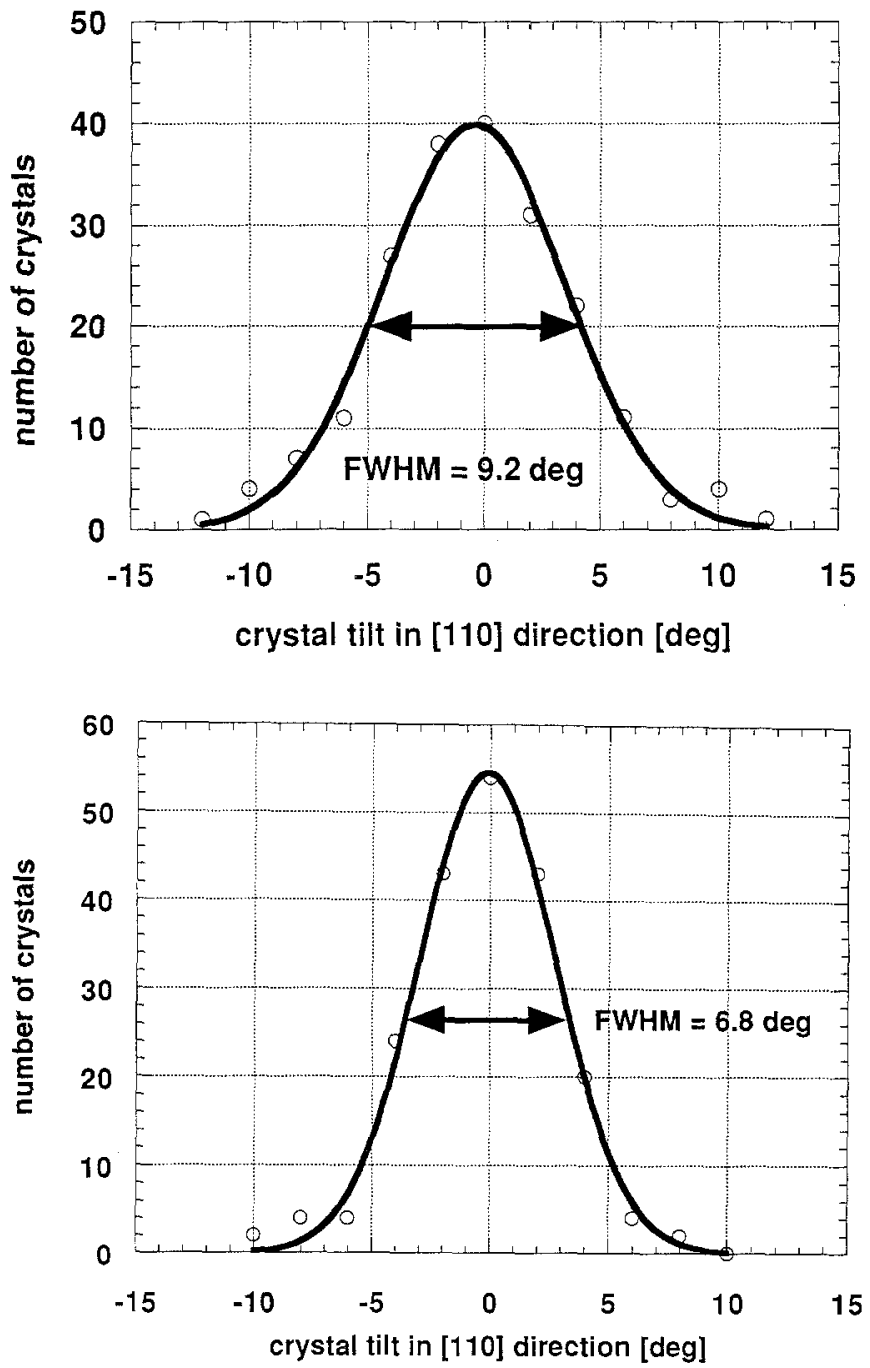

(c) and (d): tilt for sample 2 in [100] and [110] direction. Mean width of tilt distribution for sample 1 is $\pm 4.3^{\circ}$, while for sample 2 it is reduced to $\pm 3.4^{\circ}$ 
It should read:

Besides the good macroscopic crystal morphology a statistical tilt up to $\pm 4.3^{\circ}$ of the oriented crystallites has been found relative to the silicon substrates.

\section{Page 20:}

Sample 1 has a FWHM of $10.4^{\circ}$, while sample 2 results in a FWHM of $8.0^{\circ}$.

It should read:

Sample 1 has a FWHM of $8.6^{\circ}$, while sample 2 results in a FWHM of $6.7^{\circ}$.
Page 22:

It has been found that, besides the good macroscopical crystal morphology, a statistical tilting of the oriented crystallites exists up to $\pm 5.2^{\circ}$ relative to the silicon substrates.

It should read:

It has been found that, besides the good macroscopic crystal morphology, a statistical tilting of the oriented crystallites exists up to $\pm 4.3^{\circ}$ relative to the silicon substrates.

Figure $5 \mathrm{a}-\mathrm{d}$ should be replaced by the figure on page. 